\title{
Catalytic, Enantioselective Formal Synthesis of Monoterpene Indole
}

\author{
Alkaloid (-)-Alstoscholarine \\ Jian-Neng Yao, Xiao Liang, Kun Wei and Yu-Rong Yang* \\ State Key Laboratory of Phytochemistry and Plant Resources in West China, \\ Kunming Institute of Botany, Chinese Academy of Sciences, Kunming 650201, China \\ yangyurong@mail.kib.ac.cn
}

\section{Table of Contents}

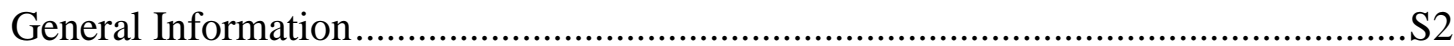

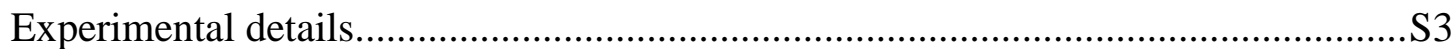

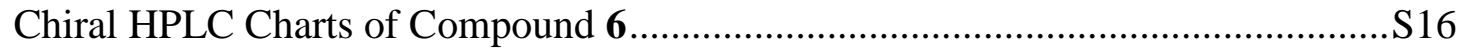

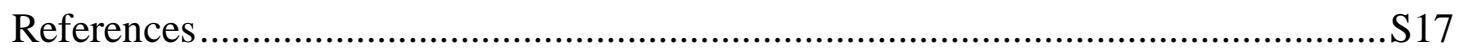

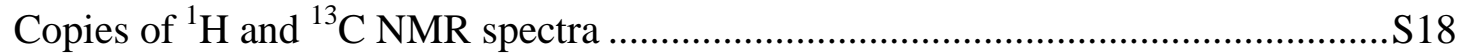




\section{General Information}

Unless otherwise stated, all oxygen or moisture sensitive reactions were conducted in flame-dried glassware under an atmosphere of nitrogen. All solvents were purified and dried according to standard methods prior to use. The phosphoramidite ligands ${ }^{[1]}$, allylic alcohol $\mathbf{7}^{[2]}$, 4-((4-methoxybenzyl)oxy)butanal $\mathbf{8}^{[3]}$ were prepared according to the reported procedure. Reagents were purchased from commercial sources and were used without further purification.

Chromatographic purification of products was accomplished using forced-flow chromatography on 200-300 mesh silica gel. All reactions were monitored by thin-layer chromatography (TLC) using silica gel GF254 pre-coated plates $(0.20 \mathrm{~mm})$ and the TLC spots were visualized with UV lamp (254 $\mathrm{nm})$, or exposure to iodine vapor.

${ }^{1} \mathrm{H}$ and ${ }^{13} \mathrm{C}$ NMR spectra were recorded on Bruker Avance III 400 NMR spectrometer. Chemical shifts were given in parts per million (ppm) with reference to residual solvent signals ${ }^{1} \mathrm{H}$ NMR: $\mathrm{CDCl}_{3}$ (7.26), ${ }^{13} \mathrm{C} \mathrm{NMR}: \mathrm{CDCl}_{3}$ (77.0), Peak multiplicities were recorded as follows: $\mathrm{s}=$ singlet, $\mathrm{d}=$ doublet, $\mathrm{t}=$ triplet, $\mathrm{m}=$ multiplet, brs $=$ broad singlet. Infrared (IR) spectra were recorded on a Thermo Scientific Nicolet ${ }^{\mathrm{TM}}$ iS 10 FR-IR spectrometer. High-resolution mass spectral (HRMS) data were obtained at the mass spectrometry service operated at Agilent 6540 Q-TOF spectrometer for electrospray ionization $(\mathrm{ESI})$ and were reported as $(\mathrm{m} / \mathrm{z})$. Optical rotations $\left([\alpha]_{\mathrm{D}}\right)$ were measured on an Autopol VI polarimeter. Melting points were measured on a WRX-5A melting point apparatus. High-performance liquid chromatography (HPLC) analysis was performed on an Agilent 1260 series system using Daicel Chiralpak ${ }^{\circledR}$ AD-H column $(5 \mu \mathrm{m}$ particle size, $4.6 \mathrm{~mm} \times 250 \mathrm{~mm})$ with $n$-hexane and $i$-PrOH as mobile phases. 


\section{Optimization of Dual Catalytic Allylation}

\section{General procedure for optimization study}<smiles>C=CC(O)c1cn(C(=O)OC(C)(C)C)c2ccccc12</smiles>

7

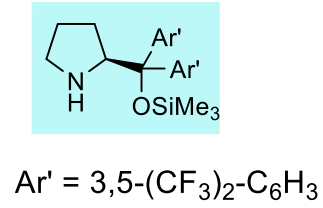

(S)-A

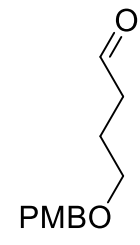

8

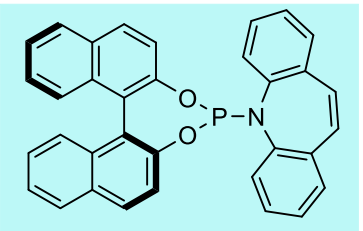

(S)-L

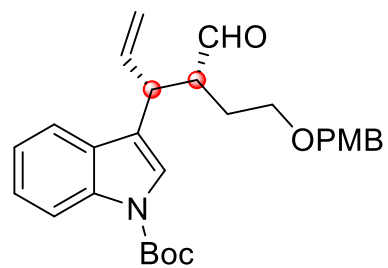

6

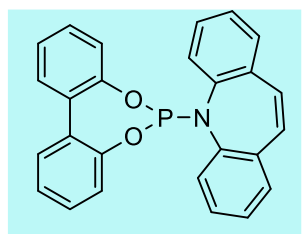

achiral L

$\left[\{\operatorname{Ir}(\operatorname{cod}) \mathrm{Cl}\}_{2}\right](2.7 \mathrm{mg}, 0.004 \mathrm{mmol}, 0.04$ equiv. $)$ and $(\boldsymbol{S})-\mathbf{L}(8.1 \mathrm{mg}, 0.016 \mathrm{mmol}$, 0.16 equiv.) were added to a nitrogen filled dry flask. Then anhydrous 1,2-dichloroethane $(330 \mu \mathrm{L})$ was added and the solution was vigorously stirred for 15 min. To the resulting dark red solution was sequentially added allylic alcohol 7 (27.3 mg, $0.1 \mathrm{mmol}, 1.0$ equiv.), aldehyde 8 (41.6 mg, $0.2 \mathrm{mmol}, 2.0$ equiv.), amine catalyst (S)-A (9.0 mg, $0.015 \mathrm{mmol}, 0.15$ equiv.) and promoter. The resulting solution was stirred at room temperature for the amount of time indicated and monitored by TLC. Upon complete consumption of the starting material, the reaction was filtered through a short pad of silica gel with petroleum ether/ethyl acetate (1:1), concentrated in vacuo, purified by silica gel column chromatography (petroleum ether/ethyl acetate 15:1) to give the desired product. The product diastereomer was determined by the ${ }^{1} \mathrm{H}$ NMR spectrum of the crude mixture. Racemic sample of $\mathbf{6}$ was obtained by using the above procedure with amine catalyst DL-proline and achiral ligand $\mathbf{L}$ instead of $(\boldsymbol{S})$-A and $(\boldsymbol{S})$-L, respectively. Enantiomeric excess was determined by HPLC analysis (Chiralpak AD-H).

Table S1 Optimization of Dual Catalytic Allylation of Indole-based Allylic Alcohol ${ }^{a}$ 


\begin{tabular}{|c|c|c|c|c|}
\hline entry & promoter $(\mathrm{mol} \%)$ & $\operatorname{yield}^{b}(\%)^{b}$ & $\mathrm{dr}^{c}$ & $\mathrm{ee}^{d}(\%)$ \\
\hline 1 & $\mathrm{Zn}(\mathrm{OTf})_{2}(10)$ & 75 & $1.8: 1$ & 97 \\
\hline 2 & $\mathrm{Sc}(\mathrm{OTf})_{3}(10)$ & 31 & $9: 1$ & $>99$ \\
\hline 3 & $\mathrm{Yb}(\mathrm{OTf})_{3}(10)$ & 60 & $2.8: 1$ & 98 \\
\hline 4 & $\mathrm{PhCO}_{2} \mathrm{H}(100)$ & $25(33)$ & $4.2: 1$ & 98 \\
\hline 5 & $\mathrm{~F}_{3} \mathrm{CCO}_{2} \mathrm{H}(50)$ & 66 & $2.6: 1$ & 89 \\
\hline 6 & $\mathrm{CCl}_{3} \mathrm{CO}_{2} \mathrm{H}(75)$ & 64 & $5: 1$ & 98 \\
\hline 7 & $\mathrm{CHCl}_{2} \mathrm{CO}_{2} \mathrm{H}(75)$ & 76 & $2: 1$ & 96 \\
\hline 8 & Maleic acid (75) & 74 & $9: 1$ & $>99$ \\
\hline $9^{f}$ & Maleic acid (75) & 74 & $9: 1$ & $>99$ \\
\hline \multicolumn{5}{|c|}{$\begin{array}{l}{ }^{a} \text { Reactions were run on a } 0.1 \mathrm{mmol} \text { scale under the standard } \\
\text { conditions. }{ }^{b} \text { Isolated yield, yields refer to the diastereomeric } \\
\text { mixture of isolated products, based on the recovered starting } \\
\text { material in parentheses. }{ }^{c} \text { The } \mathrm{dr} \text { was determined by }{ }^{1} \mathrm{H} \mathrm{NMR} \\
\text { analysis of the crude reaction mixtures. }{ }^{d} \text { Enantiomeric excess } \\
\text { was determined by chiral HPLC analysis. }{ }^{f} \text { Reaction was run a } \\
2.0 \text { mmol scale. }\end{array}$} \\
\hline
\end{tabular}

\section{Experimental Procedures}

\section{Synthesis of compound 6}<smiles>C=CC(O)c1cn(C(=O)OCc2ccccc2)c2ccccc12</smiles>

7

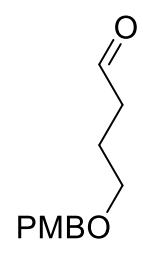

8<smiles>C=C[C@@H](OCCOC)O[C@@H](C=O)c1cn(C(=O)OC(C)(C)C)c2ccccc12</smiles>

$6 \operatorname{dr} 9: 1$, ee $>99 \%$

$\left[\{\operatorname{Ir}(\operatorname{cod}) \mathrm{Cl}\}_{2}\right](54.0 \mathrm{mg}, 0.08 \mathrm{mmol}, 0.04$ equiv. $)$ and $(\boldsymbol{S})-\mathbf{L}(162.0 \mathrm{mg}, 0.32$ mmol, 0.16 equiv.) were added to a nitrogen filled dry flask. Then anhydrous 1,2-dichloroethane $(4.0 \mathrm{~mL})$ was added and the solution was vigorously stirred for $15 \mathrm{~min}$. To the resulting dark red solution was sequentially added allylic alcohol 7 (546.0 mg, 2 mmol, 1.0 equiv), aldehyde 8 (832.0 mg, 4 mmol, 2.0 
equiv.), amine catalyst (S)-A (180.0 mg, $0.3 \mathrm{mmol}, 0.15$ equiv.) and maleic acid (174.0 mg, $1.5 \mathrm{mmol}, 0.75$ equiv.). The resulting solution was stirred for $3 \mathrm{~h}$ at room temperature and monitored by TLC. Upon complete consumption of the starting material, the reaction was filtered through a short pad of silica gel with petroleum ether/ethyl acetate (1:1), concentrated in vacuo, purified by silica gel column chromatography (petroleum ether/ethyl acetate 15:1) to give compound 6 (685.0 mg, 74\% yield) as a colorless oil. $\mathrm{R}_{f}=0.50$ (petroleum ether/EtOAc 4:1); $[\alpha]_{\mathrm{D}}^{20}=+32.5\left(c=0.46, \mathrm{CHCl}_{3}\right)$; IR (ART): $v_{\max } 3109,2976,2953,2858,2724$, $1728,1611,1586,1513,1475,1452,1370,1309$, 1249, 1157, 1089, 1035, 766, $748 \mathrm{~cm}^{-1} ;{ }^{1} \mathbf{H}$ NMR $\left(400 \mathrm{MHz}, \mathrm{CDCl}_{3}\right): \delta 9.61(1 \mathrm{H}, \mathrm{d}, J=3.0 \mathrm{~Hz}), 8.12(1 \mathrm{H}, \mathrm{d}, J$ $=8.0 \mathrm{~Hz}), 7.53(1 \mathrm{H}, \mathrm{d}, J=8.0 \mathrm{~Hz}), 7.40(1 \mathrm{H}, \mathrm{s}), 7.31(1 \mathrm{H}, \mathrm{t}, J=7.5 \mathrm{~Hz}), 7.23$ $(1 \mathrm{H}, \mathrm{t}, J=7.5 \mathrm{~Hz}), 7.19(2 \mathrm{H}, \mathrm{d}, J=8.4 \mathrm{~Hz}), 6.85(2 \mathrm{H}, \mathrm{d}, J=8.4 \mathrm{~Hz}), 5.95(1 \mathrm{H}$, ddd, $J=17.2,9.5,9.5 \mathrm{~Hz}), 5.21-5.15(2 \mathrm{H}, \mathrm{m}), 4.35(2 \mathrm{H}, \mathrm{m}), 3.90(1 \mathrm{H}, \mathrm{t}, J=8.4$ Hz), $3.79(3 \mathrm{H}, \mathrm{s}), 3.45(2 \mathrm{H}, \mathrm{m}), 2.97(1 \mathrm{H}, \mathrm{m}), 2.00(2 \mathrm{H}, \mathrm{m}), 1.66(9 \mathrm{H}, \mathrm{s}) \mathrm{ppm}$; ${ }^{13}$ C NMR (100 MHz, $\left.\mathrm{CDCl}_{3}\right): \delta$ 203.9, 159.2, 149.6, 136.8, 135.6, 130.2, 129.4, $129.3,124.6,123.1,122.5,120.2,119.3,117.4,115.4,113.7,83.7,72.6,67.5$, 55.2, 52.1, 41.3, 28.2, $27.8 \mathrm{ppm}$; HRMS(ESI): $\mathrm{m} / \mathrm{z}[\mathrm{M}+\mathrm{Na}]^{+}$calcd for $\mathrm{C}_{28} \mathrm{H}_{33} \mathrm{NO}_{5} \mathrm{Na}^{+}$486.2251, found 486.2254. $>99 \%$ ee [(Chiralpak AD-H) Hexane $/ i-\operatorname{PrOH}\left(230 \mathrm{~nm}, 25{ }^{\circ} \mathrm{C}\right)=98.5 / 1.5,0.75 \mathrm{~mL} / \mathrm{min}, \mathrm{t}_{\mathrm{R} 1}=26.04 \mathrm{~min}$ (minor), $\mathrm{t}_{\mathrm{R} 2}=30.43 \min$ (major)].<smiles>C=CO[C@H](C=O)c1cn(C(=O)OC(C)(C)C)c2ccccc12</smiles>

6

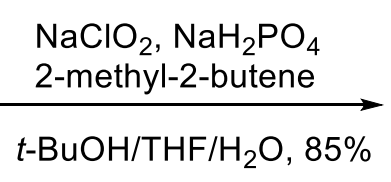

$t$-BuOH/THF/ $/ \mathrm{H}_{2} \mathrm{O}, 85 \%$

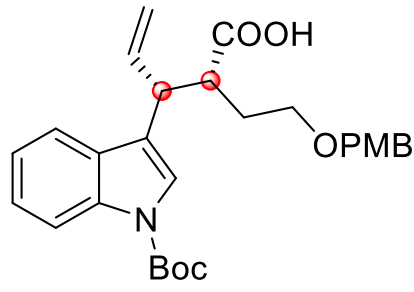

9

Synthesis of carboxylic acid 9: To a stirred solution of aldehyde 6 (1.65 g, 3.56 mmol, 1.0 equiv. $)$ in $t$-BuOH $(32 \mathrm{~mL})$ and $\mathrm{THF}(8 \mathrm{~mL})$ at room temperature, and 
2-methyl-2-butene (14.95 g, $213.6 \mathrm{mmol}, 60.0$ equiv.) was added followed by a solution of $\mathrm{NaClO}_{2}\left(3.60 \mathrm{~g}, 80 \% 32.04\right.$ mmol, 9.0 equiv.) and $\mathrm{NaH}_{2} \mathrm{PO}_{4}$ (2.99 g, 24.92 mmol, 7.0 equiv. $)$ in water $(12.5 \mathrm{~mL})$. The reaction mixture was stirred at room temperature for $2 \mathrm{~h}$. Upon complete conversion of the starting material as judged by TLC, the reaction mixture was diluted with saturated aq. $\mathrm{Na}_{2} \mathrm{~S}_{2} \mathrm{O}_{3}$ solution $(20 \mathrm{~mL})$ and extracted with EtOAc $(3 \times 60 \mathrm{~mL})$. The combined organic phase was washed with brine, dried over $\mathrm{Na}_{2} \mathrm{SO}_{4}$, filtered, concentrated in vacuo and purified by silica gel column chromatography (petroleum ether/EtOAc 3:1) to give carboxylic acid $\mathbf{9}$ (1.45 $\mathrm{g}, 85 \%$ yield) as a light yellow oil. $\mathrm{R}_{f}=0.22$ (petroleum ether/ EtOAc 2:1). $[\alpha]_{\mathrm{D}}^{20}=$ $+28.8\left(c=0.43, \mathrm{CHCl}_{3}\right)$; IR (ART): $v_{\max } 3109,3074,2975,2932,2866,1732,1705$, 1638,1612 , 1513, 1475, 1452, 1421, 1307, 1250, 1220, 1158, 1125, 1081, 1035, 922, 841, 764, $748 \mathrm{~cm}^{-1} ;{ }^{1} \mathbf{H}$ NMR (400 MHz, $\left.\mathrm{CDCl}_{3}\right): \delta 8.12(1 \mathrm{H}, \mathrm{m}), 7.57(1 \mathrm{H}, \mathrm{d}, J=7.8$ Hz), $7.49(1 \mathrm{H}, \mathrm{s}), 7.28(1 \mathrm{H}, \mathrm{t}, J=7.4 \mathrm{~Hz}), 7.19(1 \mathrm{H}, \mathrm{t}, J=7.4 \mathrm{~Hz}), 7.14(2 \mathrm{H}, \mathrm{d}, J=$ $8.5 \mathrm{~Hz}), 6.80(2 \mathrm{H}, \mathrm{d}, J=8.5 \mathrm{~Hz}), 5.90(1 \mathrm{H}, \mathrm{ddd}, J=17.2,9.6,9.6 \mathrm{~Hz}), 5.18-5.09$ $(2 \mathrm{H}, \mathrm{m}), 4.31(2 \mathrm{H}, \mathrm{s}), 3.93(1 \mathrm{H}, \mathrm{t}, J=8.5 \mathrm{~Hz}), 3.73(3 \mathrm{H}, \mathrm{s}), 3.47(1 \mathrm{H}, \mathrm{m}), 3.39(1 \mathrm{H}$, m), $3.08(1 \mathrm{H}, \mathrm{m}), 1.95(2 \mathrm{H}, \mathrm{m}), 1.62(9 \mathrm{H}, \mathrm{s}) \mathrm{ppm} ;{ }^{13} \mathrm{C}$ NMR (100 MHz, $\left.\mathrm{CDCl}_{3}\right): \delta$ $179.9,158.9,149.5,137.4,136.5,135.4,130.1,129.4,129.1,124.3,122.6,122.3$, $120.7,119.3,117.4,115.2,83.5,72.4,67.7,55.0,46.0,43.3,29.1,28.0$ ppm;

HRMS(ESI): $m / z[\mathrm{M}+\mathrm{Na}]^{+}$calcd for $\mathrm{C}_{28} \mathrm{H}_{33} \mathrm{NO}_{6} \mathrm{Na}^{+}$502.2200, found 502.2202.

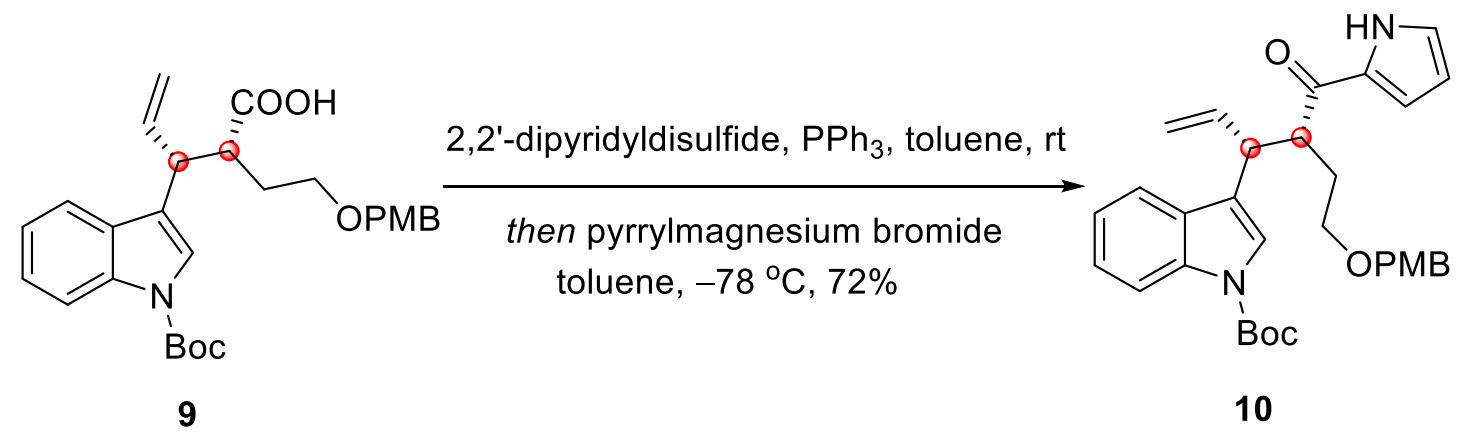

Synthesis of pyrrole 10: To a stirred solution of carboxylic acid 9 (1.54 g, $3.21 \mathrm{mmol}$, 1.0 equiv. $)$ in toluene $(10.6 \mathrm{~mL})$ under argon at room temperature was added 2,2'-dipyridyl disulfide (1060.4 mg, $4.82 \mathrm{mmol}, 1.5$ equiv.) and triphenyl phosphine 
(1262.8 mg, $4.82 \mathrm{mmol}, 1.5$ equiv.). The resulting mixture was stirred for $6 \mathrm{~h}$. This solution was then cooled to $-78{ }^{\circ} \mathrm{C}$ and pyrrylmagnesium bromide, prepared by adding ethyl magnesium bromide $(12.84 \mathrm{~mL}$ of a $1.0 \mathrm{M}$ solution in THF, $12.84 \mathrm{mmol}$, 4.0 equiv.) to freshly distilled pyrrole ( $1.07 \mathrm{~mL}, 15.41 \mathrm{mmol}, 4.8$ equiv.) in toluene $\left(12.84 \mathrm{~mL}\right.$ ) at $-40{ }^{\circ} \mathrm{C}$, was added and the reaction was stirred vigorously for $3 \mathrm{~h}$ at -78 ${ }^{\circ} \mathrm{C}$. Saturated $a q . \mathrm{NH}_{4} \mathrm{Cl}$ solution was added and the mixture was extracted with EtOAc $(3 \times 40 \mathrm{~mL})$. The combined organic phase was successively washed with saturated aq. $\mathrm{NaHCO}_{3}$, water and brine, dried over $\mathrm{Na}_{2} \mathrm{SO}_{4}$ and concentrated in vacuo. This oil was purified by flash column chromatography with petroleum ether/EtOAc $(15: 1 \rightarrow 4: 1)$ to give the title compound $\mathbf{1 0}$ as a yellow oil $(1.22 \mathrm{~g}, 72 \%$ yield $) . \mathrm{R}_{f}=$ 0.42 (petroleum ether/EtOAc 2.5:1). $[\alpha]_{\mathrm{D}}^{20}=+49.6\left(c=0.19, \mathrm{CHCl}_{3}\right)$; IR (ART): $v_{\max }$ 3259, 3135, 3074, 2959, 2918, 2871, 2849, 1729, 1632, 1613, 1586, 1544, 1512, 1473, 1452, 1429, 1406, 1368, 1307, 1256, 1156, 1093, 1080, 1019, 921, 857, 806, 764, 603 $\mathrm{cm}^{-1}$; ${ }^{1} \mathbf{H}$ NMR: (400 MHz, $\left.\mathrm{CDCl}_{3}\right) \delta 9.55(1 \mathrm{H}, \mathrm{brs}), 8.03(1 \mathrm{H}, \mathrm{d}, J=7.8 \mathrm{~Hz}), 7.60$ $(1 \mathrm{H}, \mathrm{d}, J=7.8 \mathrm{~Hz}), 7.36(1 \mathrm{H}, \mathrm{s}), 7.24(1 \mathrm{H}, \mathrm{t}, J=7.4 \mathrm{~Hz}), 7.19(1 \mathrm{H}, \mathrm{t}, J=7.4 \mathrm{~Hz})$, $7.15(2 \mathrm{H}, \mathrm{d}, J=8.3 \mathrm{~Hz}), 6.97-6.95(1 \mathrm{H}, \mathrm{m}), 6.84-6.80(3 \mathrm{H}, \mathrm{m}), 6.18-6.16(1 \mathrm{H}$, m), $5.96(1 \mathrm{H}, \mathrm{ddd}, J=17.0,9.6,9.6 \mathrm{~Hz}), 5.27-5.10(2 \mathrm{H}, \mathrm{m}), 4.26(2 \mathrm{H}, \mathrm{s}), 4.00(1 \mathrm{H}$, $\mathrm{t}, J=9.3 \mathrm{~Hz}), 3.86(1 \mathrm{H}, \mathrm{td}, J=9.9,3.4 \mathrm{~Hz}), 3.78(3 \mathrm{H}, \mathrm{s}), 3.46-3.42(1 \mathrm{H}, \mathrm{m}), 3.34-$ $3.28(1 \mathrm{H}, \mathrm{m}), 2.16-2.13(1 \mathrm{H}, \mathrm{m}), 2.07-2.02(1 \mathrm{H}, \mathrm{m}), 1.56(9 \mathrm{H}, \mathrm{s}) \mathrm{ppm} ;{ }^{13} \mathbf{C}$ NMR: $\left(100 \mathrm{MHz}, \mathrm{CDCl}_{3}\right) \delta 192.5,159.0,149.5,138.2,133.1,130.4,130.4,129.7,129.3$, $124.9,124.2,122.6,122.2,121.4,119.5,116.9,116.7,115.1,113.6,110.6,83.2,72.5$, 67.8, 55.2, 46.5, 43.8, 31.4, $28.1 \mathrm{ppm} ;$ HRMS(ESI): $\mathrm{m} / \mathrm{z}[\mathrm{M}+\mathrm{Na}]^{+}$calcd for $\mathrm{C}_{32} \mathrm{H}_{36} \mathrm{~N}_{2} \mathrm{O}_{5} \mathrm{Na}^{+}$551.2516, found 551.2519.

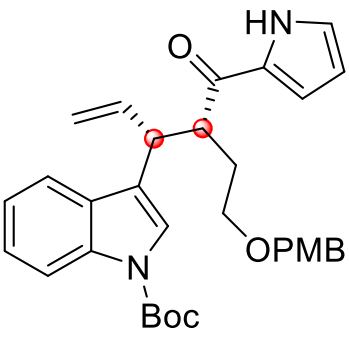

10
1. $\mathrm{OsO}_{4}, \mathrm{NMO}, \mathrm{NaIO}_{4}$

2. $\mathrm{NaClO}_{2}, \mathrm{NaH}_{2} \mathrm{PO}_{4}$ 2-methyl-2-butene

3. $\mathrm{TMSCHN}_{2}, \mathrm{CH}_{3} \mathrm{OH}$ $66 \%$ overall

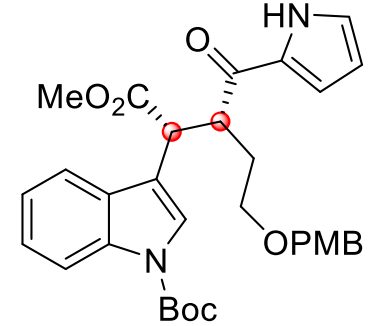

5 
Synthesis of ester 5: To a solution of compound 10 (320.0 mg, $0.60 \mathrm{mmol}, 1.0$ equiv.) in acetone $(3.36 \mathrm{~mL})$ and $\mathrm{H}_{2} \mathrm{O}(0.84 \mathrm{~mL})$ was added $\mathrm{OsO}_{4}\left(300.0 \mu \mathrm{L}, 0.06 \mathrm{M}\right.$ in $\mathrm{H}_{2} \mathrm{O}$, 0.018 mmol, 0.03 equiv.) and $\mathrm{NMO}\left(0.3 \mathrm{~mL}, 4.8 \mathrm{M}\right.$ in $\mathrm{H}_{2} \mathrm{O}, 1.44 \mathrm{mmol}, 2.4$ equiv.). The mixture was stirred at room temperature for $48 \mathrm{~h}$ and then quenched with saturated aq. $\mathrm{Na}_{2} \mathrm{~S}_{2} \mathrm{O}_{3}(15 \mathrm{~mL})$. The solution was extracted with EtOAc $(5 \times 30 \mathrm{~mL})$. The combined organic layers were washed with brine, dried over $\mathrm{Na}_{2} \mathrm{SO}_{4}$ and concentrated in vacuo. The residue was then dissolved in $\mathrm{MeOH}(4.0 \mathrm{~mL})$ and a solution of $\mathrm{NaIO}_{4}\left(192.6 \mathrm{mg}, 0.9 \mathrm{mmol}, 1.5\right.$ equiv.) in $\mathrm{H}_{2} \mathrm{O}(2.0 \mathrm{~mL})$ was added. The reaction mixture was stirred at room temperature for $30 \mathrm{~min}$. The solution was diluted with water and extracted with EtOAc $(3 \times 15 \mathrm{~mL})$. The combined organic layers were washed with brine, dried over $\mathrm{Na}_{2} \mathrm{SO}_{4}$ and concentrated in vacuo. This crude aldehyde was dissolved in $t$-BuOH $(4.8 \mathrm{~mL})$ and THF $(1.2 \mathrm{~mL})$ and then was added 2-methyl-2-butene (2.52 g, $36.0 \mathrm{mmol}, 60.0$ equiv.). To this reaction mixture was added a solution of $\mathrm{NaClO}_{2}\left(607.5 \mathrm{mg}, 80 \%, 5.4 \mathrm{mmol}, 9.0\right.$ equiv.) and $\mathrm{NaH}_{2} \mathrm{PO}_{4}$ (504.0 mg, $4.2 \mathrm{mmol}, 7.0$ equiv.) in $\mathrm{H}_{2} \mathrm{O}(2.1 \mathrm{~mL})$. The reaction mixture was stirred at room temperature for $1 \mathrm{~h}$. After complete conversion of the starting material as judged by TLC, the reaction mixture was extracted with EtOAc $(3 \times 30 \mathrm{~mL})$. The combined organic phase was washed with brine, dried over $\mathrm{Na}_{2} \mathrm{SO}_{4}$, and concentrated in vacuo. This crude carboxylic acid was dissolved in $\mathrm{MeOH}(3.0 \mathrm{~mL})$ and $\mathrm{TMSCHN}_{2}(1.5 \mathrm{~mL}$ of a 2.0 M solution in hexane, $3.0 \mathrm{mmol}, 5.0$ equiv.) was added. The reaction mixture was stirred at room temperature for $30 \mathrm{~min}$. TLC analysis indicated no starting material and silica gel ( $6.0 \mathrm{~g})$ was added to the reaction mixture to consume unreacted reagent. The slurry was stirred for 15 min and then filtered by washing with EtOAc (120.0 mL). The filtrate was concentrated in vacuo. The crude residue was purified by column chromatography (petroleum ether/EtOAc $5: 1 \rightarrow 3: 1$ ) to give methyl ester 5 $\left(221.8 \mathrm{mg}, 66 \%\right.$ yield) as a colorless oil. $\mathrm{R}_{f}=0.26$ (petroleum ether/EtOAc $\left.3: 1\right) .[\alpha]_{\mathrm{D}}^{20}$ $=-35.1\left(c=0.15, \mathrm{CHCl}_{3}\right)$; IR (ART): $v_{\max } 3268,3134,3052,2981,2952,2929,2851$, 1733, 1635, 1613, 1586, 1567, 1544, 1513, 1474, 1452, 1431, 1407, 1368, 1330, 1308, 1250, 1155, 1088, 763, 749, $603 \mathrm{~cm}^{-1} ;{ }^{1} \mathbf{H} \mathbf{~ N M R}\left(400 \mathrm{MHz}, \mathrm{CDCl}_{3}\right): \delta 9.53$ (1H, brs), $8.02(1 \mathrm{H}, \mathrm{d}, J=7.7 \mathrm{~Hz}), 7.71(1 \mathrm{H}, \mathrm{d}, J=6.9 \mathrm{~Hz}), 7.51(1 \mathrm{H}, \mathrm{s}), 7.26-7.22(2 \mathrm{H}, \mathrm{m})$, 
$7.14(2 \mathrm{H}, \mathrm{d}, J=8.2 \mathrm{~Hz}), 6.97(1 \mathrm{H}, \mathrm{d}, J=3.4 \mathrm{~Hz}), 6.83(2 \mathrm{H}, \mathrm{d}, J=8.2 \mathrm{~Hz}), 6.75(1 \mathrm{H}$, d, $J=3.1 \mathrm{~Hz}), 6.15-6.12(1 \mathrm{H}, \mathrm{m}), 4.34(1 \mathrm{H}, \mathrm{d}, J=10.8 \mathrm{~Hz}), 4.25(2 \mathrm{H}, \mathrm{s}), 4.24-$ $4.15(1 \mathrm{H}, \mathrm{m}), 3.78(3 \mathrm{H}, \mathrm{s}), 3.66(3 \mathrm{H}, \mathrm{s}), 3.45-3.34(2 \mathrm{H}, \mathrm{m}), 2.18-2.12(1 \mathrm{H}, \mathrm{m})$, 2.08 - 2.02 (1H, m), 1.56 (9H, s) ppm; ${ }^{13} \mathbf{C}$ NMR (100 MHz, $\left.\mathrm{CDCl}_{3}\right): \delta$ 190.7, 172.9, $159.0,149.3,135.1,132.8,130.3,129.3,129.2,125.3,124.4,124.4,122.5,119.5$, 117.3, 116.2, 115.0, 113.6, 110.7, 83.5, 72.4, 67.4, 55.2, 52.2, 45.6, 44.8, 32.2, 28.0 ppm; HRMS(ESI): $m / z[\mathrm{M}+\mathrm{Na}]^{+}$calcd for $\mathrm{C}_{32} \mathrm{H}_{36} \mathrm{~N}_{2} \mathrm{O}_{7} \mathrm{Na}^{+}$583.2415, found 583.2411.
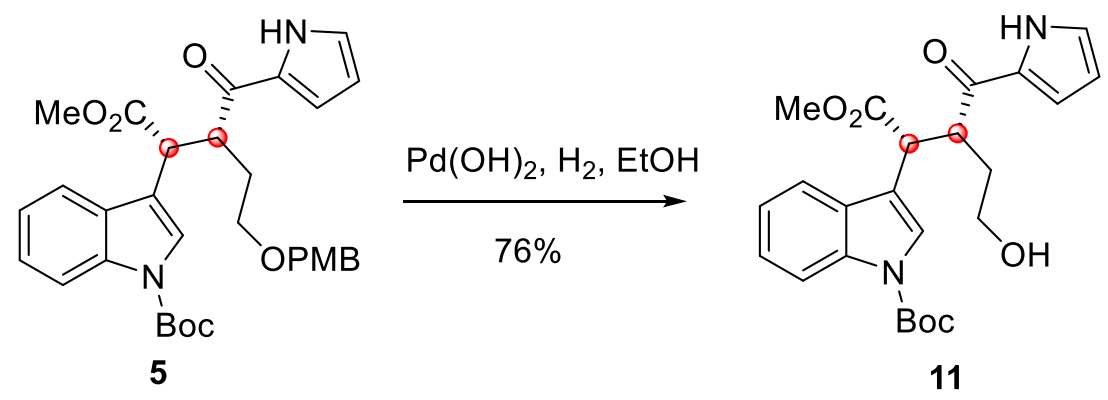

Synthesis of alcohol 11: To a solution of methyl ester 5 (198.0 mg, $0.35 \mathrm{mmol}, 1.0$ equiv.) in $\mathrm{EtOH}(3.5 \mathrm{~mL})$ was added $\mathrm{Pd}(\mathrm{OH})_{2}(49.1 \mathrm{mg}, 0.07 \mathrm{mmol}, 0.2$ equiv., palladium hydroxide $20 \%$ on carbon, wetted with ca. $50 \%$ water) at room temperature. The system was then evacuated and refilled with $\mathrm{H}_{2}$ three times. After stirring $48 \mathrm{~h}$ at room temperature under atmospheric pressure of $\mathrm{H}_{2}$, the reaction was filtered through a short pad of silica gel with EtOAc $(100 \mathrm{~mL})$, concentrated in vacuo. The resulting crude residue was purified by silica gel column chromatography (petroleum ether/EtOAc 5:1) to afford alcohol $11(117.0 \mathrm{mg}, 76 \%)$ as a colorless oil. $\mathrm{R}_{f}=0.40$ (petroleum ether/EtOAc 1:1), $[\alpha]_{\mathrm{D}}^{20}=-46.5\left(c=0.23, \mathrm{CHCl}_{3}\right)$; IR $(\mathrm{ART}): v_{\max } 3283$, 3137, 3084, 3054, 2977, 2951, 2885, 2849, 1732, 1626, 1545, 1452, 1432, 1368, 1339, 1309, 1256, 1194, 1152, 1114, 1047, 1018, 761, $746 \mathrm{~cm}^{-1} ;{ }^{\mathbf{1}} \mathbf{H}$ NMR (400 MHz, $\left.\mathrm{CDCl}_{3}\right): \delta 9.51(1 \mathrm{H}, \mathrm{brs}), 8.02(1 \mathrm{H}, \mathrm{d}, J=6.0 \mathrm{~Hz}), 7.72(1 \mathrm{H}, \mathrm{d}, J=7.1 \mathrm{~Hz}), 7.51(1 \mathrm{H}$, s), $7.26-7.20(2 \mathrm{H}, \mathrm{m}), 7.01(1 \mathrm{H}, \mathrm{s}), 6.82(1 \mathrm{H}, \mathrm{s}), 6.16(1 \mathrm{H}, \mathrm{d}, J=2.8 \mathrm{~Hz}), 4.37(1 \mathrm{H}, \mathrm{d}$, $J=10.3 \mathrm{~Hz}), 4.24(1 \mathrm{H}, \mathrm{ddd}, J=10.9,10.3,3.5 \mathrm{~Hz}), 3.69(3 \mathrm{H}, \mathrm{s}), 3.65-3.59(2 \mathrm{H}, \mathrm{m})$, $2.15-2.09(1 \mathrm{H}, \mathrm{m}), 1.98-1.95(1 \mathrm{H}, \mathrm{m}), 1.57(9 \mathrm{H}, \mathrm{s}) \mathrm{ppm} ;{ }^{13} \mathbf{C} \mathbf{N M R}(100 \mathrm{MHz}$, 
$\left.\mathrm{CDCl}_{3}\right): \delta 190.9,173.2,149.3,135.1,132.7,129.3,125.7,124.5,124.4,122.6,119.5$, $117.4, \quad 116.2, \quad 115.0, \quad 110.9, \quad 83.6,60.2, \quad 52.3, \quad 45.3,44.6, \quad 35.0,28.1$ ppm; HRMS(ESI): $m / z[\mathrm{M}+\mathrm{Na}]^{+}$calcd for $\mathrm{C}_{24} \mathrm{H}_{28} \mathrm{~N}_{2} \mathrm{O}_{6} \mathrm{Na}^{+} 463.1840$, found 463.1841 .
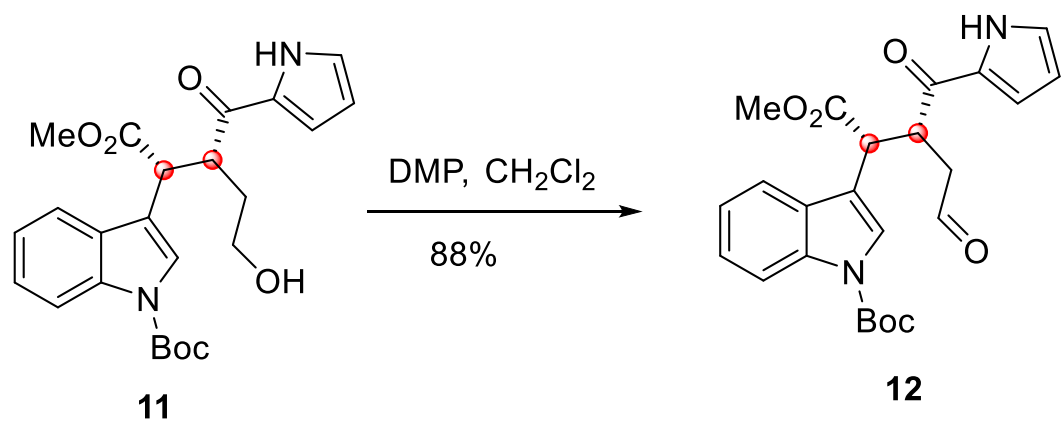

Synthesis of aldehyde 12: To a solution alcohol 11 (73.0 mg, $0.16 \mathrm{mmol}, 1.0$ equiv.) in $\mathrm{CH}_{2} \mathrm{Cl}_{2}(1.6 \mathrm{~mL})$ was added Dess-Martin periodinane (DMP; $135.7 \mathrm{mg}, 0.32 \mathrm{mmol}$, 2 equiv.). After stirring for $30 \mathrm{~min}$, the reaction was quenched with saturated $a q$. $\mathrm{Na}_{2} \mathrm{~S}_{2} \mathrm{O}_{3}(5 \mathrm{~mL})$ and diluted with $\mathrm{CH}_{2} \mathrm{Cl}_{2}(8 \mathrm{~mL})$. The layers were separated and the aqueous layer was extracted with $\mathrm{CH}_{2} \mathrm{Cl}_{2}(3 \times 5 \mathrm{~mL})$. The organic layers were combined, dried over $\mathrm{Na}_{2} \mathrm{SO}_{4}$, and evaporated in vacuo. The crude residue was purified via silica gel column chromatography (petroleum ether/EtOAc 3:1) to afford the corresponding aldehyde $12(62.0 \mathrm{mg}, 88 \%$ yield $)$ as a colorless oil. $\mathrm{R}_{f}=0.24$ (petroleum ether/EtOAc 2:1), $[\alpha]_{\mathrm{D}}^{20}=-136.1\left(c=0.26, \mathrm{CHCl}_{3}\right)$; IR (ART): $v_{\max } 3282$, 3135, 3085, 2978, 2952, 2931, 2849, 2727, 1732, 1634, 1566, 1545, 1452, 1432, 1406, $1338,1309,1271,1256,1236,1202,1155,1115,1088,1017,936,910,764,748 \mathrm{~cm}^{-1}$; ${ }^{1}$ H NMR $\left(400 \mathrm{MHz}, \mathrm{CDCl}_{3}\right): \delta 9.72(1 \mathrm{H}, \mathrm{s}), 9.18(1 \mathrm{H}, \mathrm{brs}), 8.04(1 \mathrm{H}, \mathrm{d}, J=7.8 \mathrm{~Hz})$, $7.65(1 \mathrm{H}, \mathrm{d}, J=7.8 \mathrm{~Hz}), 7.56(1 \mathrm{H}, \mathrm{s}), 7.29-7.22(2 \mathrm{H}, \mathrm{m}), 6.96(1 \mathrm{H}, \mathrm{dd}, J=4.0,2.3$ $\mathrm{Hz}), 6.89(1 \mathrm{H}, \mathrm{t}, J=2.8 \mathrm{~Hz}), 6.18-6.16(1 \mathrm{H}, \mathrm{m}), 4.45(1 \mathrm{H}, \mathrm{td}, J=9.6,4.1 \mathrm{~Hz}), 4.33$ $(1 \mathrm{H}, \mathrm{d}, J=9.6 \mathrm{~Hz}), 3.69(3 \mathrm{H}, \mathrm{s}), 3.14(1 \mathrm{H}, \mathrm{dd}, J=18.0,8.4 \mathrm{~Hz}), 2.88(1 \mathrm{H}, \mathrm{dd}, J=$ 18.0, 4.1 Hz), 1.62 (9H, s) ppm; ${ }^{13} \mathbf{C}$ NMR (100 MHz, $\left.\mathrm{CDCl}_{3}\right): \delta$ 199.2, 189.0, 172.3, $149.3,135.2$, 131.4, 129.0, 125.5, 124.7, 124.6, 122.7, 119.1, 117.5, 115.3, 115.2, 111.0, 83.8, 52.4, 44.9, 44.2, 42.7, $28.1 \mathrm{ppm} ; \mathbf{H R M S ( E S I ) : m / z ~ [ M - H ] ~ c a l c d ~ f o r ~}$ $\mathrm{C}_{24} \mathrm{H}_{26} \mathrm{~N}_{2} \mathrm{O}_{6}^{-}$437.1718, found 437.1719. 


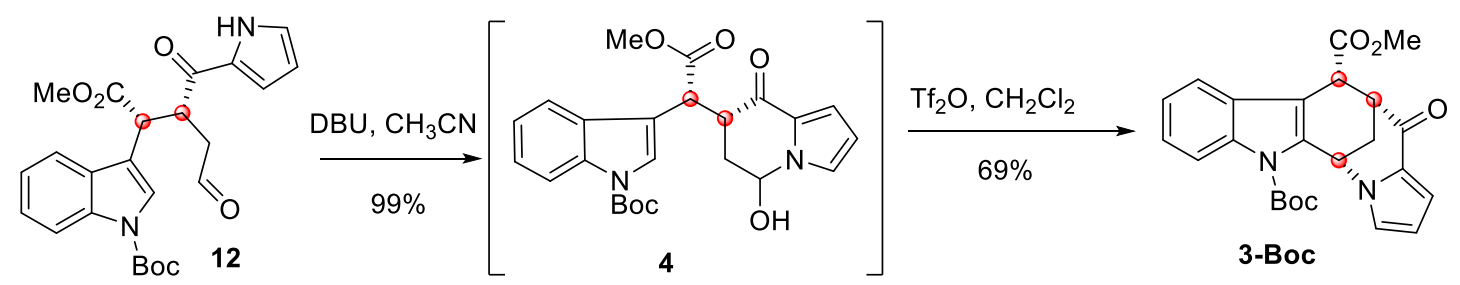

Synthesis of pentacycle 3-Boc: To a solution of aldehyde $12(57.0 \mathrm{mg}, 0.13 \mathrm{mmol}$, 1.0 equiv.) in anhydrous $\mathrm{CH}_{3} \mathrm{CN} \quad(2.6 \quad \mathrm{~mL})$ was added 1,8-diazabicyclo[5.4.0]undec-7-ene (DBU; $1.9 \mu \mathrm{L}, 0.013 \mathrm{mmol}, 0.1$ equiv.). The reaction mixture was stirred at room temperature for $1 \mathrm{~h}$. After complete conversion of the starting material as judged by TLC, the reaction mixture was evaporated in vасио. The crude residue was filtered through a short pad of silica gel (petroleum ether/EtOAc 2:1) to afford the corresponding tetracyclic hemiaminal 4. This hemiaminal was dissolved in dry $\mathrm{CH}_{2} \mathrm{Cl}_{2}(2.6 \mathrm{~mL})$ and trifluoromethanesulfonic anhydride $\left(\mathrm{Tf}_{2} \mathrm{O}, 4.4 \mu \mathrm{L}, 0.026 \mathrm{mmol}, 0.2\right.$ equiv.) was added. The resulting mixture was stirred at $0{ }^{\circ} \mathrm{C}$ for $1 \mathrm{~h}$ and then warmed to room temperature. The mixture was stirred for $3 \mathrm{~h}$ and monitored by TLC. The mixture was then quenched with saturated aq. $\mathrm{NaHCO}_{3}(3.0 \mathrm{~mL})$ and extracted with EtOAc $(3 \times 8 \mathrm{~mL})$. The combined organic phases were washed with brine, dried over $\mathrm{Na}_{2} \mathrm{SO}_{4}$, filtered and concentrated in vacuo. The crude residue was purified by column chromatography (petroleum ether/EtOAc $5: 1 \rightarrow 1.5: 1)$ to give pentacycle 3 -Boc $\left(37.6 \mathrm{mg}, 69 \%\right.$ yield) as colorless oil. $\mathrm{R}_{f}=0.36$ (petroleum ether/EtOAc 1.5:1), $[\alpha]_{\mathrm{D}}^{20}=+261.4\left(c=0.20, \mathrm{CHCl}_{3}\right)$; IR (ART): $v_{\max }$ 3138, 3120, 3016, 2977, 2947, 1751, 1733, 1646, 1526, 1477, 1452, 1433, 1420, 1392 , 1365, 1333, 1310, 1303, 1289, 1254, 1219, 1198, 1172, 1156, 1068, 1056, 928, 902, 880, 765, 749, $643 \mathrm{~cm}^{-1} ;{ }^{1} \mathbf{H}$ NMR $\left(400 \mathrm{MHz}, \mathrm{CDCl}_{3}\right): \delta 7.95(1 \mathrm{H}, \mathrm{d}, J=8.4 \mathrm{~Hz})$, $7.47(1 \mathrm{H}, \mathrm{s}), 7.29(1 \mathrm{H}, \mathrm{t}, J=7.6 \mathrm{~Hz}), 7.24-7.21(1 \mathrm{H}, \mathrm{m}), 7.02(1 \mathrm{H}, \mathrm{m}), 6.44(1 \mathrm{H}, \mathrm{m})$, $6.16(1 \mathrm{H}, \mathrm{m}), 4.30(1 \mathrm{H}, \mathrm{d}, J=6.7 \mathrm{~Hz}), 3.74(3 \mathrm{H}, \mathrm{s}), 3.47-3.45(1 \mathrm{H}, \mathrm{m}), 2.92-2.87$ $(1 \mathrm{H}, \mathrm{dt}, J=13.3,3.5 \mathrm{~Hz}), 2.52(1 \mathrm{H}, \mathrm{d}, J=13.3 \mathrm{~Hz}), 1.74(9 \mathrm{H}, \mathrm{s}) \mathrm{ppm} ;{ }^{13} \mathbf{C}$ NMR $(100$ $\left.\mathrm{MHz}, \mathrm{CDCl}_{3}\right): \delta 186.3,171.5,150.6,135.5,135.2,128.7,127.6,126.6,125.2,123.1$, $119.5,116.2,116.1,114.2,110.1,85.0,52.3,46.1,43.9,42.2,33.5,28.3$ ppm; HRMS(ESI): $m / z[\mathrm{M}+\mathrm{Na}]^{+}$calcd for $\mathrm{C}_{24} \mathrm{H}_{24} \mathrm{~N}_{2} \mathrm{O}_{5} \mathrm{Na}^{+} \quad 443.1577$, found 437.1573. 
<smiles></smiles>

3-Boc

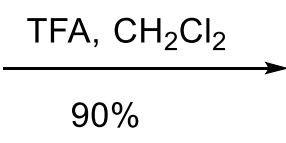

$90 \%$

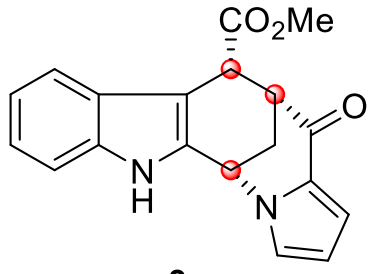

3

Synthesis of pentacyclic intermediate 3: Trifluoroacetic acid (TFA, $100 \mu \mathrm{L}$ ) was added cautiously to a stirred solution of pentacycle $3-B$ Boc $(13.0 \mathrm{mg}, 0.026 \mathrm{mmol})$ in $\mathrm{CH}_{2} \mathrm{Cl}_{2}(200 \mu \mathrm{L})$ at $0{ }^{\circ} \mathrm{C}$. The reaction mixture was stirred for $5 \mathrm{~h}$ at $\mathrm{rt}$ and then was quenched with saturated $a q \cdot \mathrm{NaHCO}_{3}(3 \mathrm{~mL})$ and extracted with EtOAc $(3 \times 5 \mathrm{~mL})$. The combined organic phases were washed with brine, dried over $\mathrm{Na}_{2} \mathrm{SO}_{4}$, filtered and concentrated in vacuo. The crude residue was purified by column chromatography (petroleum ether/EtOAc 1.5:1) to give the title product 3 (9.0 mg, 90\% yield) as a white power. $\mathrm{R}_{f}=0.12$ (petroleum ether/EtOAc 1.5:1), $[\alpha]_{\mathrm{D}}^{20}=+60.0(c=$ 0.42, $\mathrm{CHCl}_{3}$ ); m.p.: $175-176{ }^{\circ} \mathrm{C}$; IR (ART): $v_{\max } 3324,2926,2905,2849,1724,1635$, 1526, 1454, 1413, 1396, 1338, 1321, 1257, 1010, 984, 790, 700, 685, 660, $610 \mathrm{~cm}^{-1}$; ${ }^{1} \mathbf{H}$ NMR $\left(400 \mathrm{MHz}, \mathrm{CDCl}_{3}\right): \delta 8.83(1 \mathrm{H}, \mathrm{brs}), 7.29(1 \mathrm{H}, \mathrm{d}, J=7.8 \mathrm{~Hz}), 7.23(1 \mathrm{H}, \mathrm{d}, J$ $=7.8 \mathrm{~Hz}), 7.13(1 \mathrm{H}, \mathrm{t}, J=7.5 \mathrm{~Hz}), 7.06(1 \mathrm{H}, \mathrm{t}, J=7.5 \mathrm{~Hz}), 6.72(1 \mathrm{H}, \mathrm{s}), 6.67(1 \mathrm{H}, \mathrm{d}, J$ $=3.4 \mathrm{~Hz}), 5.88-5.86(1 \mathrm{H}, \mathrm{m}), 4.95(1 \mathrm{H}, \mathrm{s}), 4.32(1 \mathrm{H}, \mathrm{d}, J=6.2 \mathrm{~Hz}), 3.80(3 \mathrm{H}, \mathrm{s})$, $3.46(1 \mathrm{H}, \mathrm{s}), 2.73-2.70(1 \mathrm{H}, \mathrm{m}), 2.39-2.36(1 \mathrm{H}, \mathrm{m}) \mathrm{ppm} ;{ }^{13} \mathbf{C}$ NMR $(100 \mathrm{MHz}$, $\left.\mathrm{CDCl}_{3}\right): \delta 187.0,173.0,135.6,133.8,128.7,125.8,125.1,122.9,120.2,119.5,116.1$, 111.7, 110.3, 105.7, 52.4, 47.1, 44.2, 43.2, $33.2 \mathrm{ppm} ; \mathbf{H R M S ( E S I ) : ~} \mathrm{m} / \mathrm{z}[\mathrm{M}+\mathrm{Na}]^{+}$ calcd for $\mathrm{C}_{19} \mathrm{H}_{16} \mathrm{~N}_{2} \mathrm{O}_{3} \mathrm{Na}^{+}$434.1053, found 434.1050.

Crystal data for compound 3: $\mathrm{C}_{19} \mathrm{H}_{16} \mathrm{~N}_{2} \mathrm{O}_{3}, M=320.34, a=9.3698(5) \AA, b=$ 16.5160(8) $\mathrm{A}, c=20.7642(11) \AA, \alpha=90^{\circ}, \beta=90^{\circ}, \gamma=90^{\circ}, V=3213.3(3) \AA^{3}, T=$ 100.(2) $\mathrm{K}$, space group $P 212121, Z=8, \mu(\mathrm{Cu} \mathrm{K \alpha})=0.741 \mathrm{~mm}^{-1}, 25885$ reflections measured, 6348 independent reflections $\left(R_{i n t}=0.0299\right)$. The final $R_{l}$ values were $0.0286(I>2 \sigma(I))$. The final $w R\left(F^{2}\right)$ values were $0.0922(I>2 \sigma(I))$. The final $R_{I}$ values were 0.0292 (all data). The final $w R\left(F^{2}\right)$ values were 0.0941 (all data). The goodness of fit on $F^{2}$ was 0.862 . Flack parameter $=-0.05(4)$. 


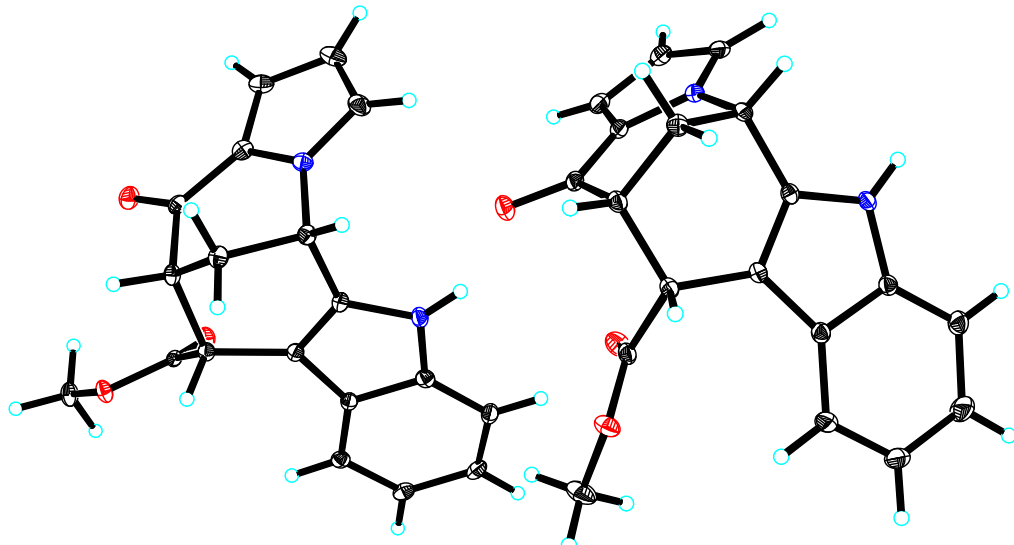

View of the molecules in an asymmetric unit.

Displacement ellipsoids are drawn at the $30 \%$ probability level.

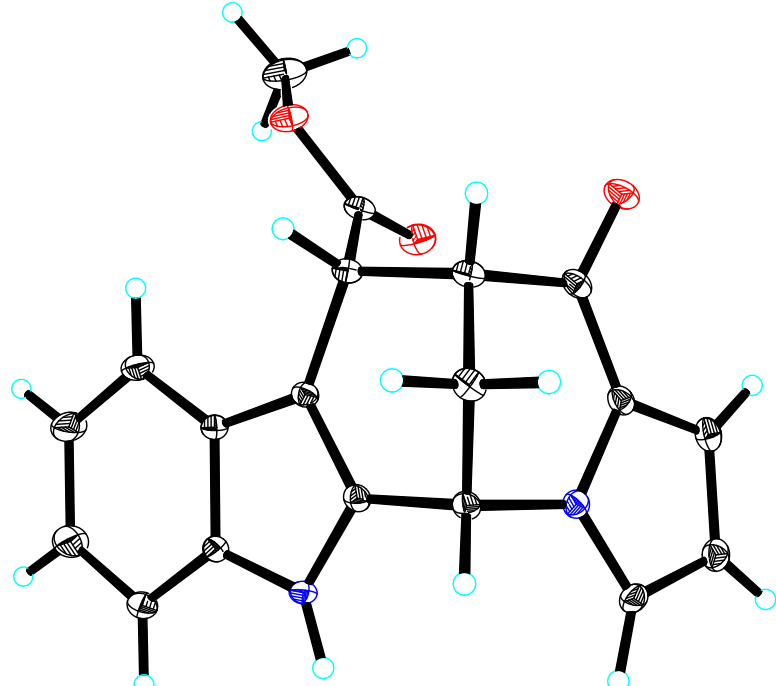

View of a molecule of compound $\mathbf{3}$ with the atom-labelling scheme.

Displacement ellipsoids are drawn at the $30 \%$ probability level. 


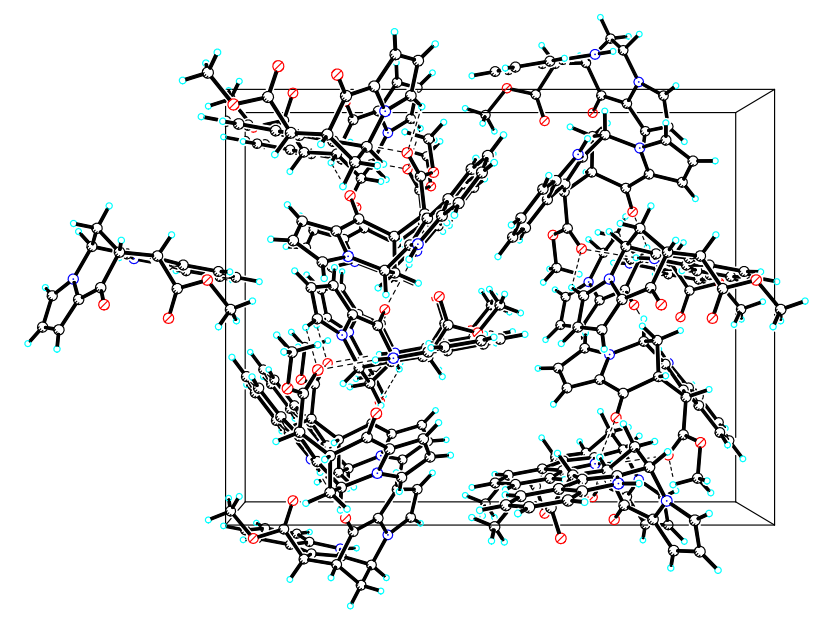

View of the pack drawing of compound 3 .

Hydrogen-bonds are shown as dashed lines.

Crystal data and structure refinement for compound $\mathbf{3}$.

Identification code

global

Empirical formula

C19 H16 N2 O3

Formula weight

320.34

Temperature

100(2) K

Wavelength

$1.54178 \AA$

Crystal system

Orthorhombic

Space group

$\mathrm{P} 22_{1} 2_{1}$

Unit cell dimensions

$\begin{array}{ll}a=9.3698(5) \AA & \alpha=90^{\circ} . \\ b=16.5160(8) \AA & \beta=90^{\circ} . \\ c=20.7642(11) \AA & \gamma=90^{\circ} .\end{array}$

Volume

3213.3(3) $\AA^{3}$

Z

8

Density (calculated)

$1.324 \mathrm{Mg} / \mathrm{m}^{3}$

Absorption coefficient

$0.741 \mathrm{~mm}^{-1}$

$\mathrm{F}(000)$

1344

Crystal size

$0.300 \times 0.220 \times 0.100 \mathrm{~mm}^{3}$

Theta range for data collection

3.42 to $72.36^{\circ}$.

Index ranges

$-11<=\mathrm{h}<=9,-20<=\mathrm{k}<=20,-25<=1<=25$

Reflections collected

25885

Independent reflections

$6348[\mathrm{R}($ int $)=0.0299]$ 
Completeness to theta $=72.36^{\circ}$

Absorption correction

Max. and min. transmission

Refinement method

Data / restraints / parameters

Goodness-of-fit on $\mathrm{F}^{2}$

Final $\mathrm{R}$ indices $[\mathrm{I}>2 \operatorname{sigma}(\mathrm{I})]$

$\mathrm{R}$ indices (all data)

Absolute structure parameter

Largest diff. peak and hole
$99.9 \%$

Semi-empirical from equivalents

0.93 and 0.82

Full-matrix least-squares on $\mathrm{F}^{2}$

6348 / 0 / 435

0.862

$\mathrm{R} 1=0.0286, \mathrm{wR} 2=0.0922$

$\mathrm{R} 1=0.0292, \mathrm{wR} 2=0.0941$

$-0.05(4)$

0.245 and -0.189 e. $\AA^{-3}$ 


\section{Chiral HPLC Charts of compound 6}

Racemate of compound $\mathbf{6}$

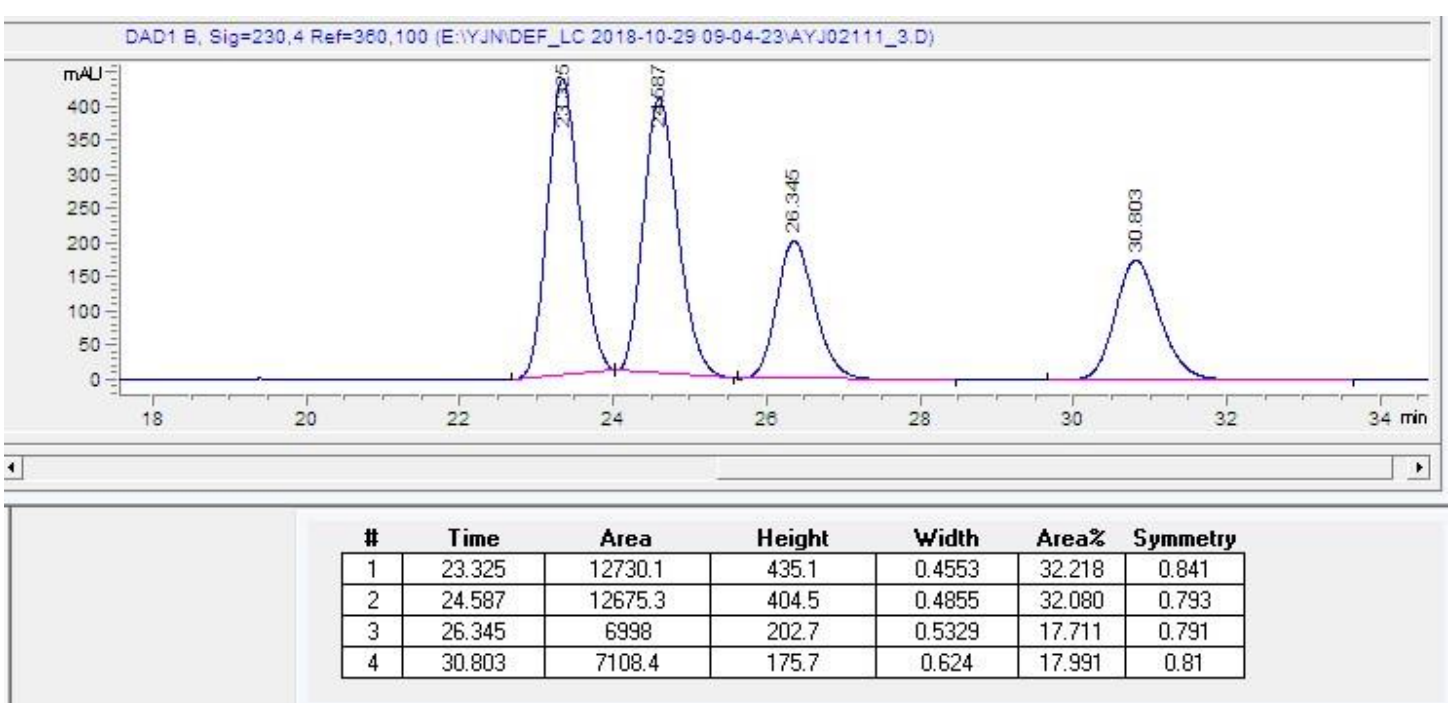

Enantioenriched mixture of compound 6

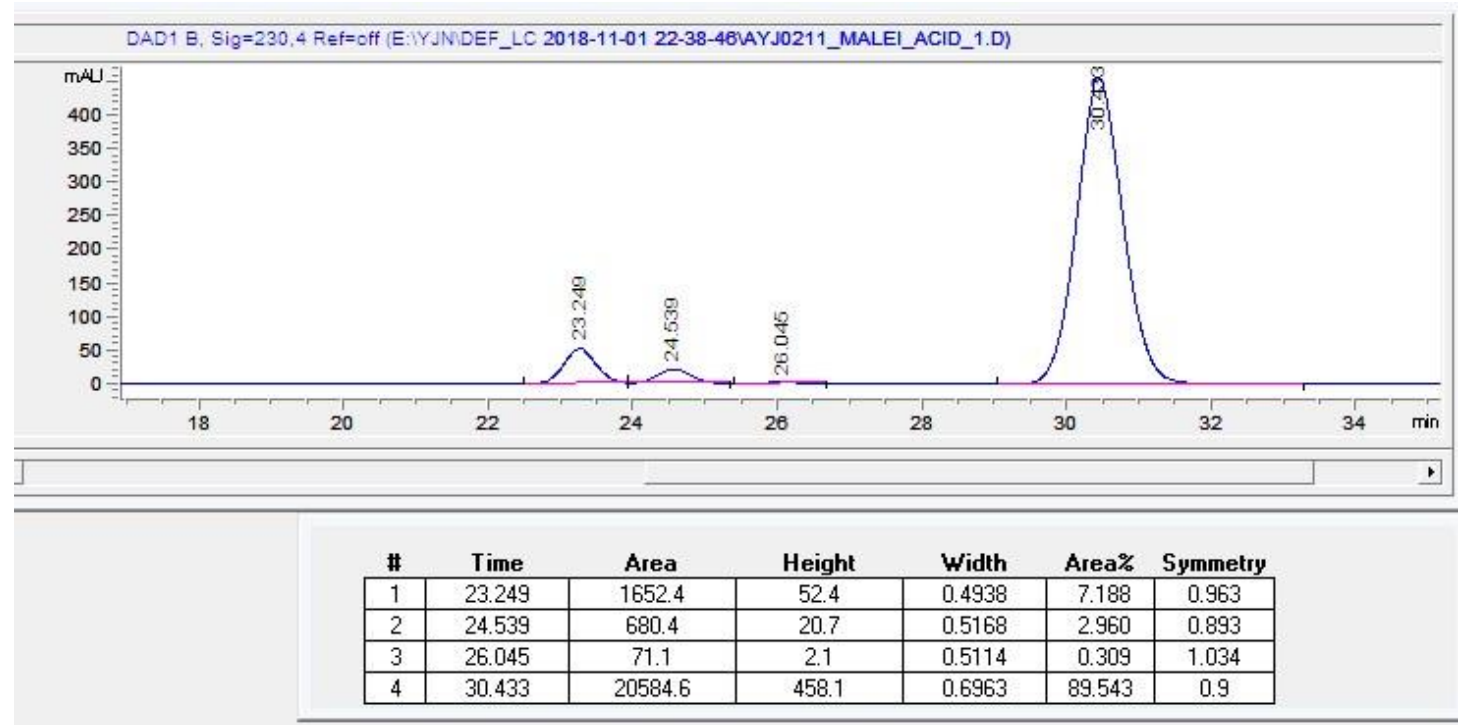




\section{References}

[1] (a) Defieber, C.; Ariger, M. A.; Moriel, P.; Carreira, E. M., Angew. Chem. Int. Ed. 2007, 46, 3139. (b) Schafroth, M. A.; Sarlah, D.; Krautwald, S.; Carreira, E. M., J. Am. Chem. Soc. 2012, 134, 20276.

[2] Liang, X.; Zhang, T. Y.; Meng, C. Y.; Li, X. D.; Wei, K.; Yang, Y. R., Org. Lett. 2018, 20, 4575.

[3] Si, C.-M.; Liu, Y.-W.; Mao, Z.-Y.; Han, P.; Du, Z.-T.; Wei, B.-G. Tetrahedron 2016, $72,8091$. 
Copies of ${ }^{1} \mathrm{H}$ and ${ }^{13} \mathrm{C}$ NMR spectra

V<smiles>C=COC(COCCOCCCC)c1cn(C(=O)OC(C)(C)C)c2ccccc12</smiles>

6

${ }^{1} \mathrm{H} \mathrm{NMR} 400 \mathrm{MHz} \mathrm{CDCl}_{3}$

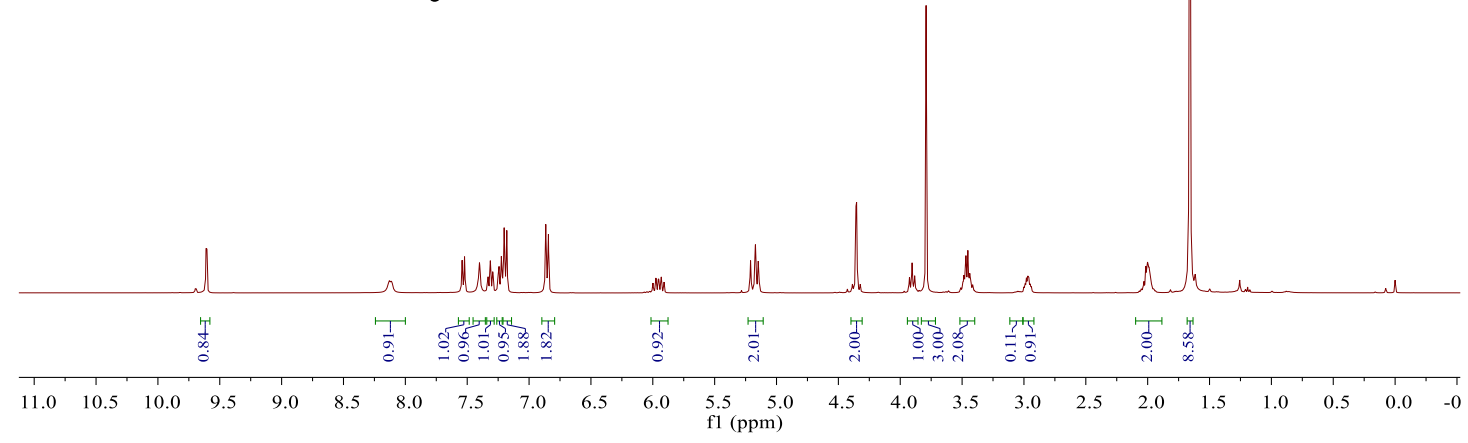

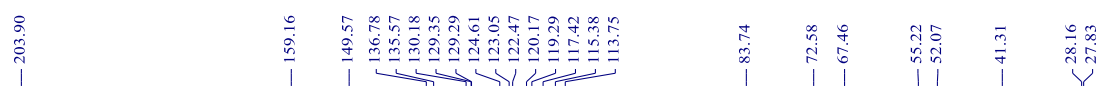<smiles>C=CO[C@@H](C=O)c1cn(C(=O)OCc2ccccc2)c2ccccc12</smiles>

6

${ }^{13} \mathrm{C} \mathrm{NMR} 100 \mathrm{MHz} \mathrm{CDCl}_{3}$

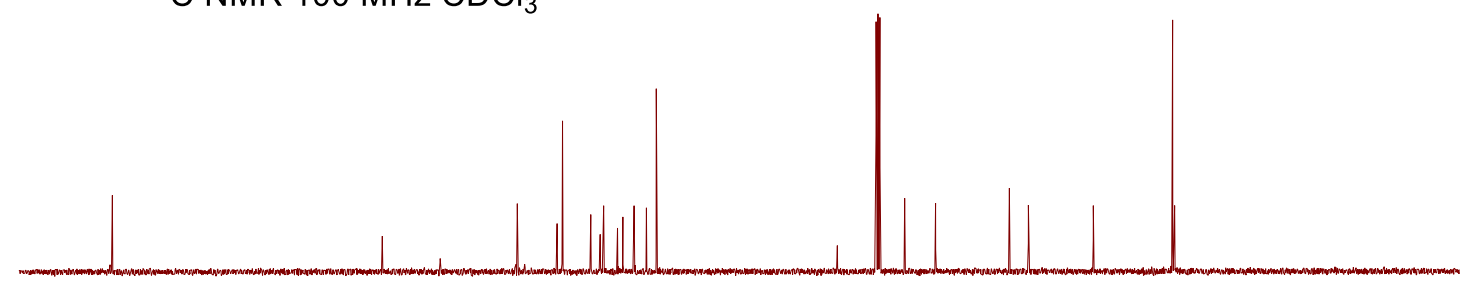

$\begin{array}{lllllllllllllllllllllllll}210 & 200 & 190 & 180 & 170 & 160 & 150 & 140 & 130 & 120 & 110 & 100 & 90 & 80 & 70 & 60 & 50 & 40 & 30 & 20 & 10 & 0 & -10\end{array}$ 
$\underbrace{20}$<smiles>[R16]OCCC(OC=C)C(=O)OCc1cn(C(=O)OCc2ccccc2)c2ccccc12</smiles>

9

${ }^{1} \mathrm{H} \mathrm{NMR} 400 \mathrm{MHz} \mathrm{CDCl}_{3}$

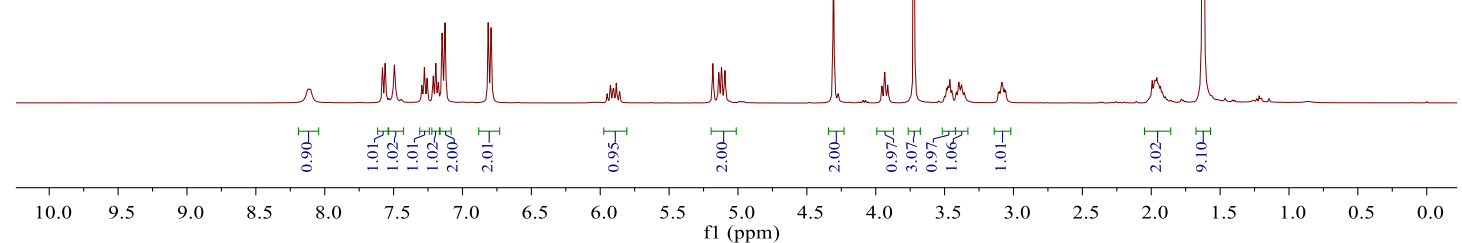

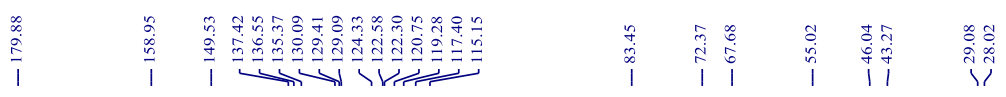<smiles>C=COC(CCO[14CH3])C(=O)OCc1cn(C(=O)OCc2ccccc2)c2ccccc12</smiles>

9

${ }^{13} \mathrm{C} \mathrm{NMR} 100 \mathrm{MHz} \mathrm{CDCl}_{3}$

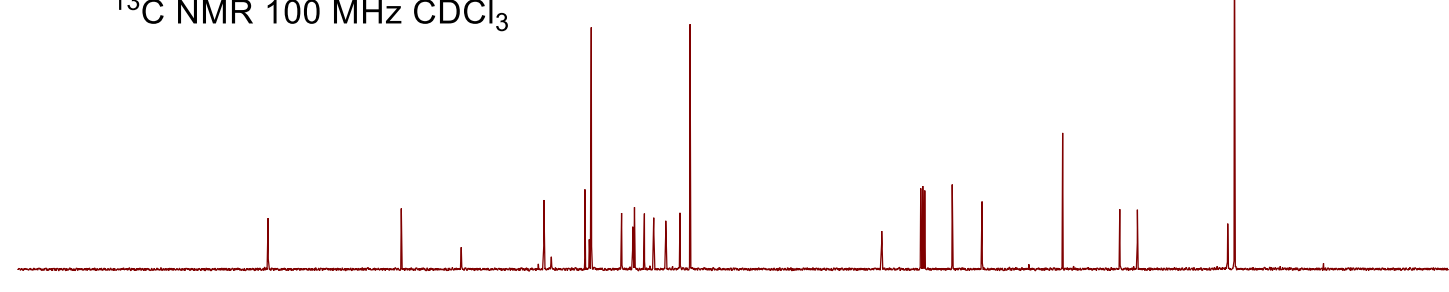

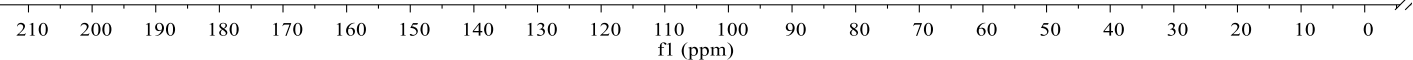


<smiles>C=COC(=O)c1cn(C(=O)OC(C)(C)C)c2ccccc12</smiles>

10

${ }^{1} \mathrm{H} \mathrm{NMR} 400 \mathrm{MHz} \mathrm{CDCl}_{3}$

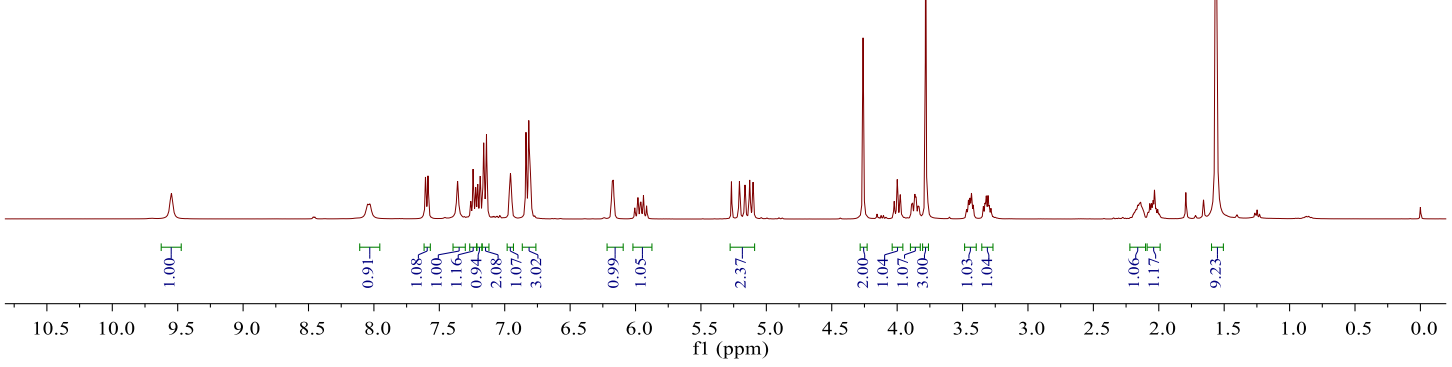

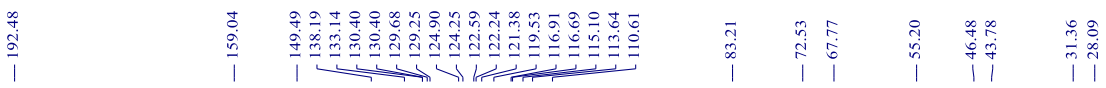<smiles>C=COC(=O)c1cn(C(=O)OCc2ccccc2)c2ccccc12</smiles>

10

${ }^{13} \mathrm{C} \mathrm{NMR} 100 \mathrm{MHz} \mathrm{CDCl}_{3}$

$\begin{array}{lllllllllllllllllllll}210 & 200 & 190 & 180 & 170 & 160 & 150 & 140 & 130 & 120 & 110 & 100 & 90 & 80 & 70 & 60 & 50 & 40 & 30 & 20 & 10\end{array}$ 


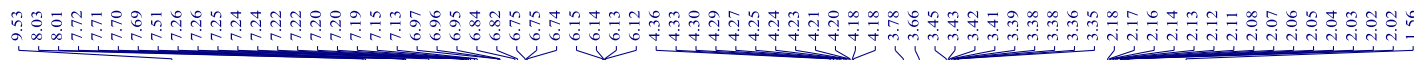<smiles>COC(=O)OCCOC(=O)c1cn(C(C)(C)C)c2ccccc12</smiles>

5

${ }^{1} \mathrm{H}$ NMR $400 \mathrm{MHz} \mathrm{CDCl}_{3}$

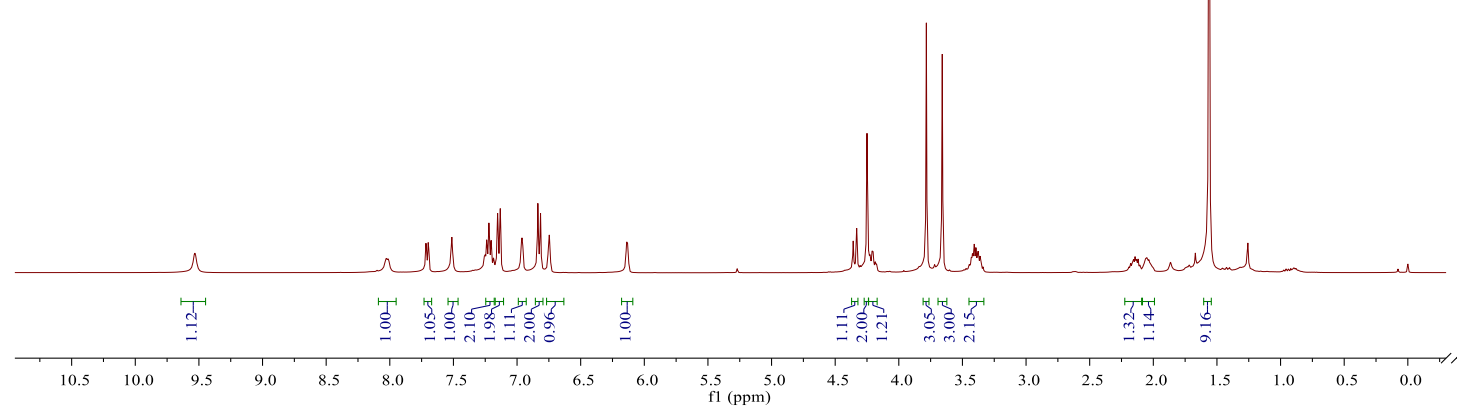

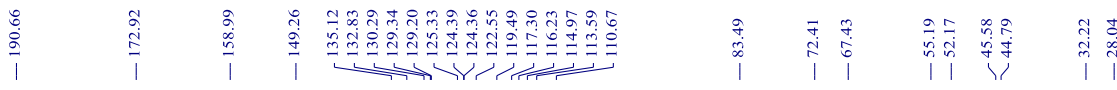<smiles>CCCCOCCOC(=O)c1cn(C(=O)OC(C)(C)C)c2ccccc12</smiles>

5

${ }^{13} \mathrm{C} \mathrm{NMR} 100 \mathrm{MHz} \mathrm{CDCl}_{3}$
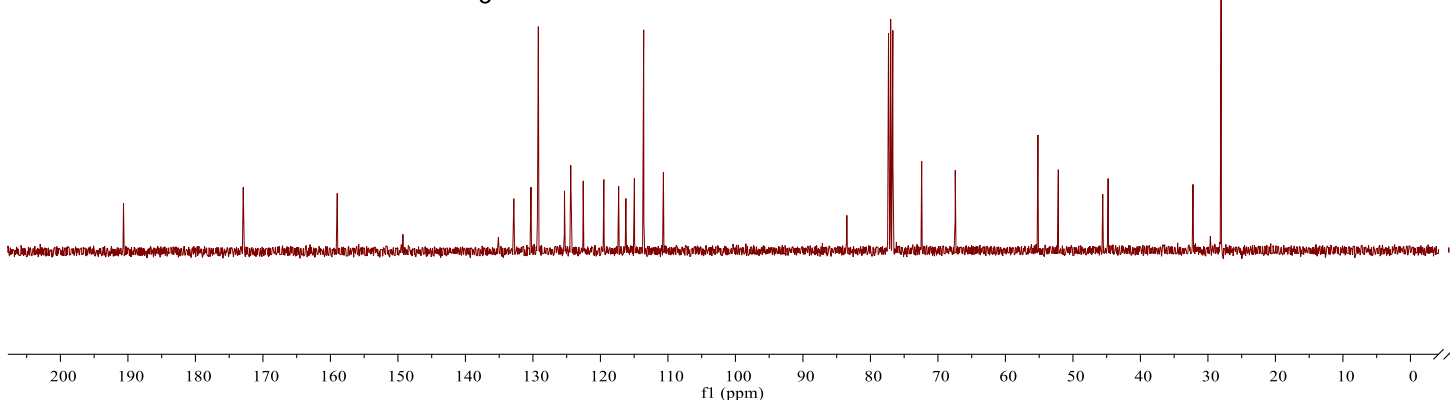
落

$\mathrm{MeO}_{2} \mathrm{C}$<smiles>CC(=O)OC(CCCO)c1cn(C(C)(C)C)c2ccccc12</smiles>

11

${ }^{1} \mathrm{H}$ NMR $400 \mathrm{MHz} \mathrm{CDCl}_{3}$

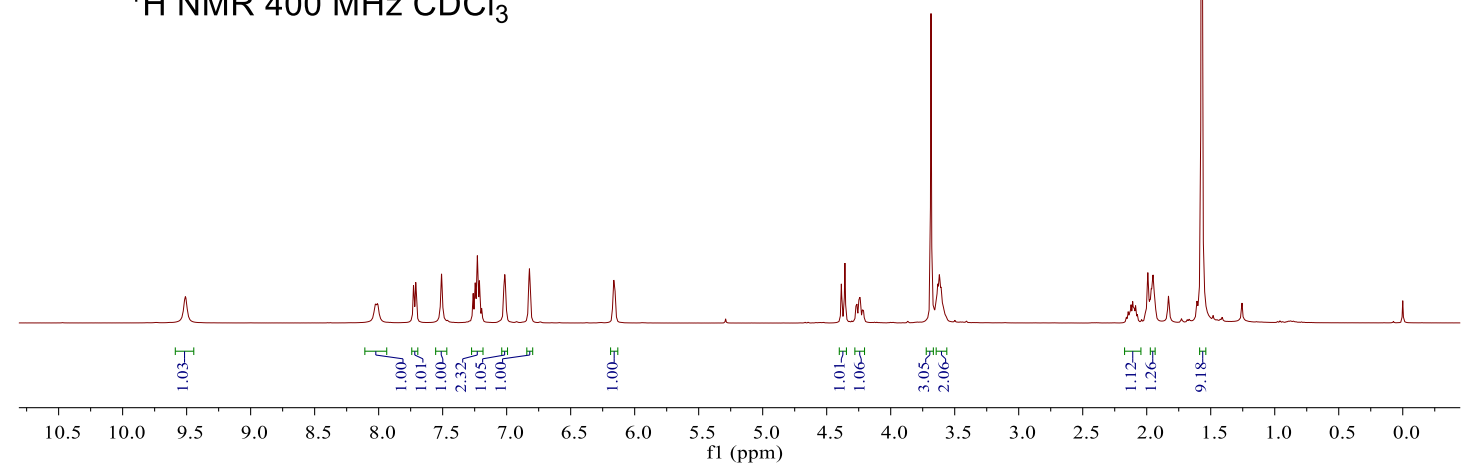

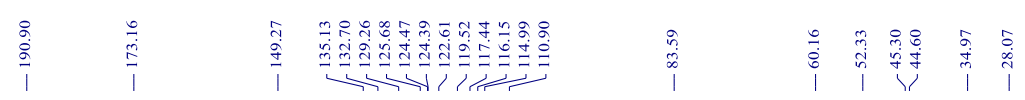<smiles>C=C(NC)C(=O)OC(=O)c1cn(C(=O)OC(C)(C)C)c2ccccc12</smiles>

11

${ }^{13} \mathrm{C} \mathrm{NMR} 100 \mathrm{MHz} \mathrm{CDCl}_{3}$

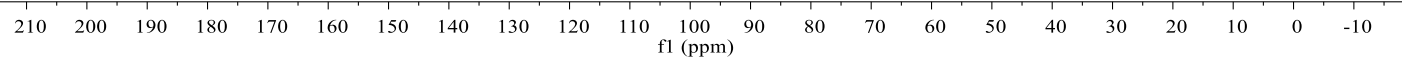




\section{告}

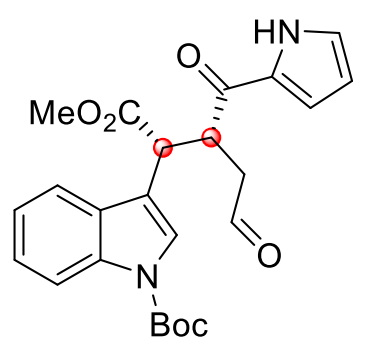

12

${ }^{1} \mathrm{H} \mathrm{NMR} 400 \mathrm{MHz} \mathrm{CDCl}_{3}$

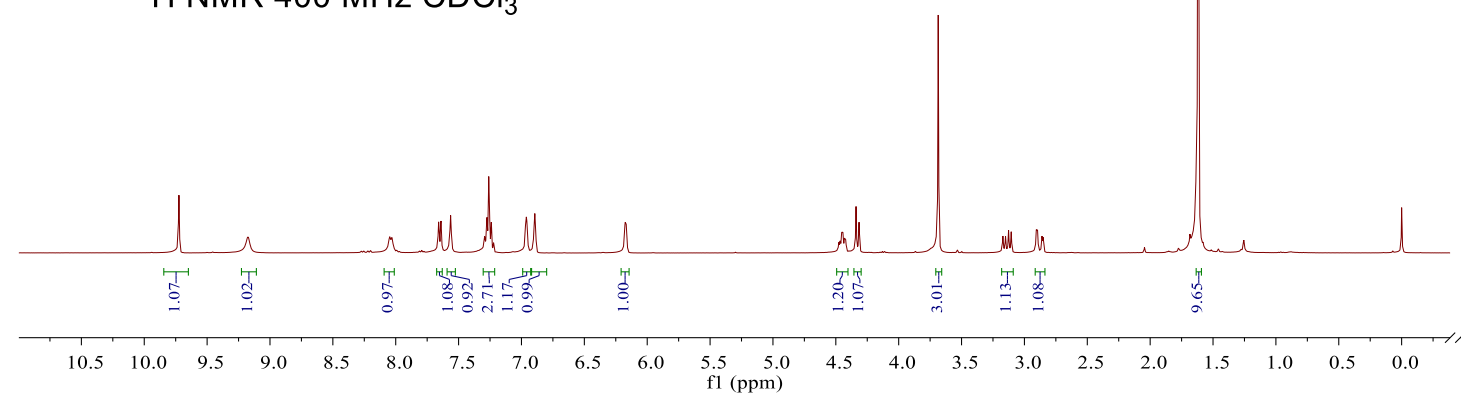

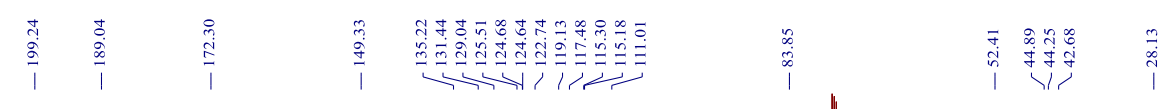<smiles>C=C(N)C(=O)OC(CC=O)c1cn(C(=O)OC(C)(C)C)c2ccccc12</smiles>

12

${ }^{13} \mathrm{C} \mathrm{NMR} 100 \mathrm{MHz} \mathrm{CDCl}_{3}$

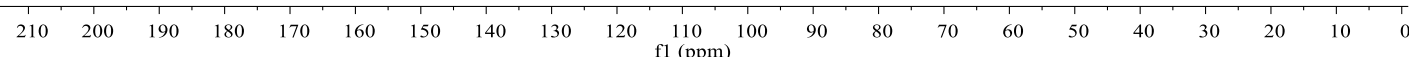




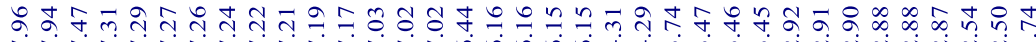

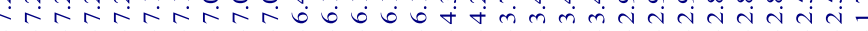<smiles></smiles>

3-Boc

${ }^{1} \mathrm{H} \mathrm{NMR} 400 \mathrm{MHz} \mathrm{CDCl}_{3}$
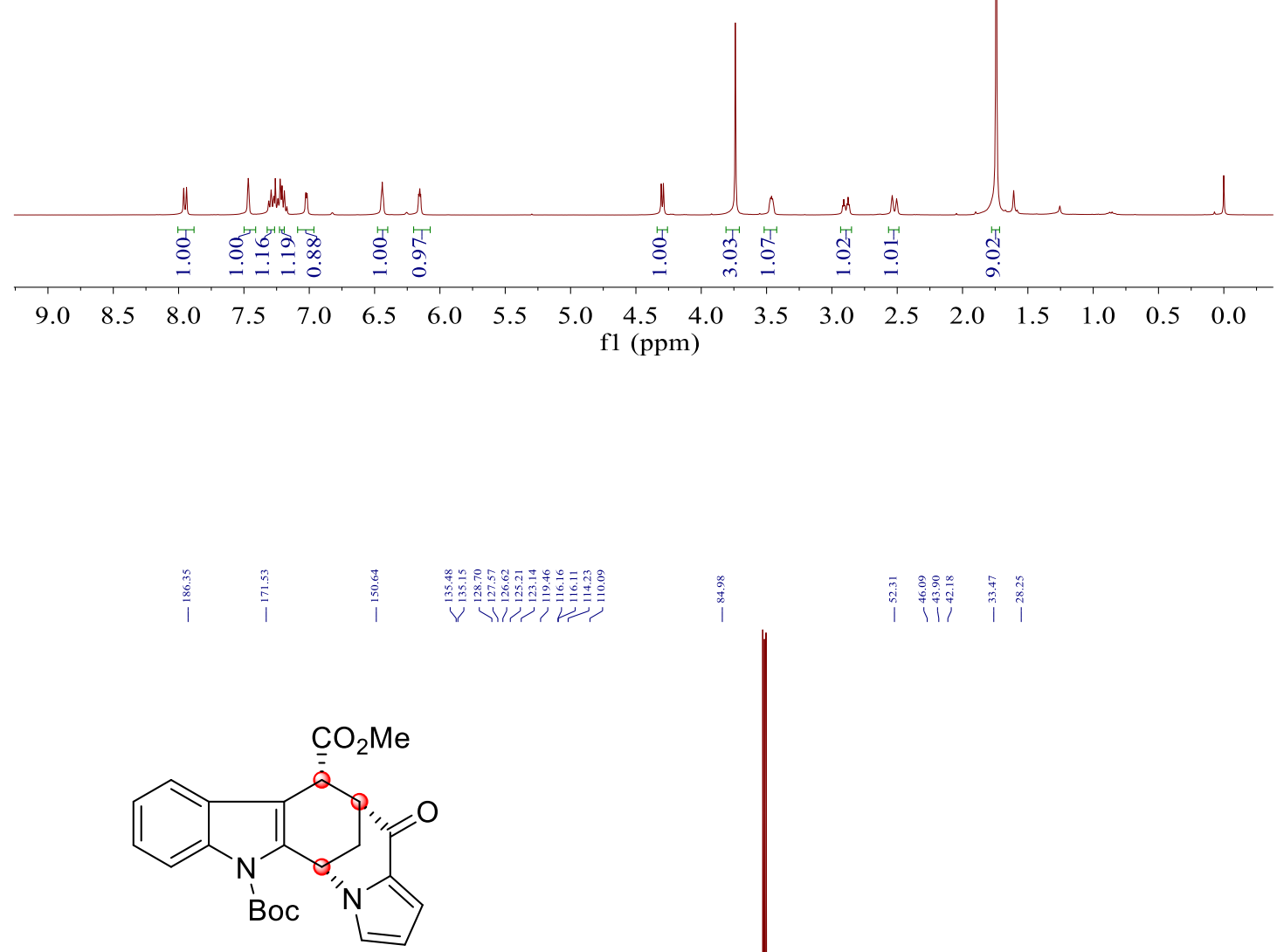

3-Boc

${ }^{13} \mathrm{C} \mathrm{NMR} \mathrm{100MHZ} \mathrm{CDCl}_{3}$

$\begin{array}{lllllllllllllllllllllll}210 & 200 & 190 & 180 & 170 & 160 & 150 & 140 & 130 & 120 & 110 & \begin{array}{c}100 \\ \mathrm{f} 1(\mathrm{ppm})\end{array} & 90 & 80 & 70 & 60 & 50 & 40 & 30 & 20 & 10 & 0 & -10\end{array}$ 


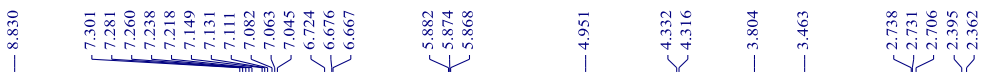<smiles></smiles>

3

${ }^{1} \mathrm{H}$ NMR $400 \mathrm{MHz} \mathrm{CDCl}_{3}$

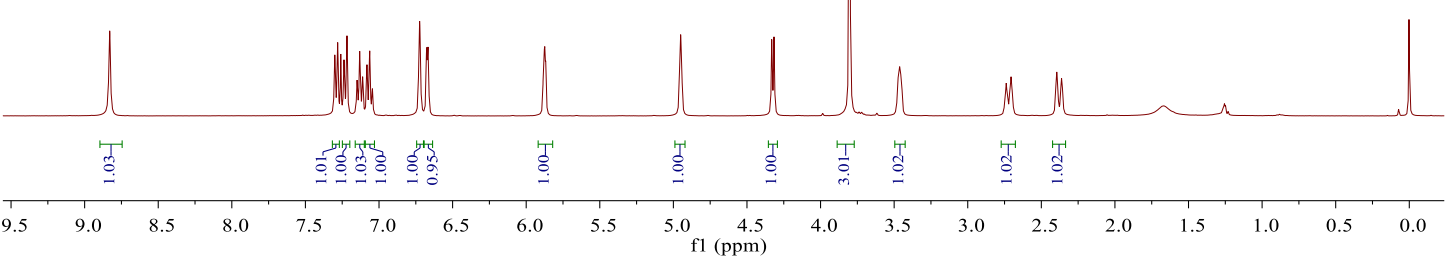

$\begin{array}{ll}n & 0 \\ 0 & 0 \\ 0 & 0 \\ 1 & 1\end{array}$

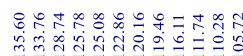

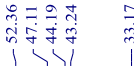

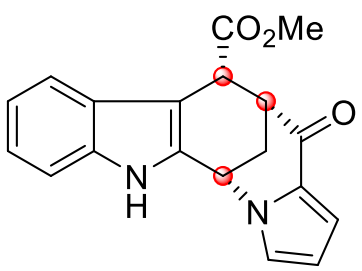

3

${ }^{13} \mathrm{C}$ NMR $100 \mathrm{MHz} \mathrm{CDCl}_{3}$

$\begin{array}{lllllllllllllllllllllll}210 & 200 & 190 & 180 & 170 & 160 & 150 & 140 & 130 & 120 & 110 \underset{\mathrm{f} 1(\mathrm{ppm})}{100} & 90 & 80 & 70 & 60 & 50 & 40 & 30 & 20 & 10 & 0 & -10\end{array}$ 